\title{
Relationships between Functional Movement Screen, VAS, ODI and Back Endurance in Individual with Nonspecific Chronic Low Back Pain
}

\author{
Kyoung-hee Noh, PT, Ph.D. ${ }^{1}$; Jae-seop Oh, PT, Ph.D. ${ }^{2}$ \\ ${ }^{1}$ Movement Training Center, Pusan, South Korea \\ ${ }^{2}$ Department of Physical Therapy, Inje University, Gimhae, South Korea
}

Background Nonspecific chronic low back pain (NCLBP) needs intervention for the management of pain and functional problems. Providing the same intervention in heterogeneous groups of subjects with NCLBP does not deliver effective back pain management. Recent studies have recommended NCLBP by classifying subjects into homogeneous groups.

Purpose The purpose of the present study is to determine whether FMS scores could evaluate the physical function of subjects with CLBP and to identify a relationship between the VAS and FMS, the ODI and FMS, and trunk endurance and FMS.

Study design Case-series study.

Methods 29 subjects with NCLBP were recruited and a relationship between trunk endurance, or VAS, or ODI and FMS was identified.

Results VAS $(r=-0.409, p<0.05)$ and the ODI $(r=0.473, p<0.01)$ were negatively correlated with the FMS. The FMS was positively correlated with the back endurance test $(r=0.381, p<0.041)$.

Conclusions FMS may assist in designing more effective individualized treatment plans to improve the functional capacity of subjects with CLBP.

Journal of KEMA 2017; 1(1): 7-12 Published Online Dec 30, 2017 pISSN: 2586-4351

Article History

Received 19 October 2017 Revised 15 November 2017 (1st)

Revised 20 November 2017 (2nd)

Accepted 20 November 2017

\section{CONTACT}

ysrehab@inje.ac.kr Jae-Seop Oh,

Department of Physical

Therapy, Inje University,

Gimhae, Republic of

Korea.

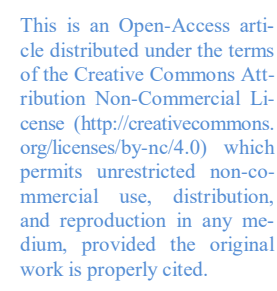

Key words Classification; Nonspecific chronic low back pain; Trunk endurance test.

IRB was approved at the Inje University Ethics

Committee for Human Investigations.

\section{INTRODUCTION}

NCLBP may result in both impaired physical functions and psychosocial problems. NCLBP subjects can experience depression, work disability, and deteriorated nociceptive sensations. ${ }^{1}$ Measures in NCLBP management may be useful in clinical decision-making and in identifying change related to the focused condition. ${ }^{2}$ However, one of the most difficult tasks associated with the management of LBP is clinical assessment. ${ }^{3}$

Some researchers have conducted self-reported questionnaires or measurement tools related to low back disability to assess daily function. ${ }^{3}$ The ODI is often used to evaluate disability related to LBP, and the VAS is a widely used method for the clinical assessment of pain intensity. ${ }^{4} \mathrm{Re}-$ search has developed to assess psychological problems and functional disabilities to make objective assessments for subjects with CLBP. ${ }^{3,5,6}$

Some researchers attempted to analyze the functional status or functional results obtained after therapeutic intervention. The back endurance test is commonly used to measure the capacity of the back muscles and trunk extensor fatigue. ${ }^{7,8}$ People with CLBP seem to have low trunk muscle strength and a low fatigue threshold. ${ }^{9}$ Generally, 
static back endurance in subjects with CLBP has been examined using the Biering-Sorenson test, the most widely used test in functional assessment. ${ }^{10,11,12}$ It often uses sit-tostand and stand-to-sit tests to assess limited hip and lumbar spine motions in subjects with LBP. ${ }^{13}$ It also uses standing on one leg to assess decreased kinetic and kinematic stability. ${ }^{14}$ Previous studies suggested a back pain scale with five tasks for the assessment of mobility-related daily activities in people with back pain ${ }^{13,14}$. Although simple function tests are commonly used for subjects with CLBP, their integrated functions regarding daily activities is not being assessed.

FMS is used as a musculoskeletal assessment method. The tasks consist of a deep squat, hurdle step, inline lunge, shoulder mobility, active straight leg raise, push-up, and rotational stability. ${ }^{2}$ FMS task performance is evaluated using standardized verbal instructions and visual inspection. FMS scores include movement conditions with or without pain and symmetrically for task performance. The primary goal of FMS is to evaluate ability a combination of physical activities such as flexibility, range of motion, muscle strength, coordination, balance, and proprioception ${ }^{2,15}$ and to predict the general risk of musculoskeletal conditions and injuries. 2,16,17 $^{2}$

In a study by Mitchell et al., there is a positive correlation between core strength and FMS ( $r=0.31, p=0.006)$ in school children. ${ }^{18}$ Deydre et al. reported a positive correlation between back endurance and FMS $(r=0.30, p=0.01)$, Ybalance test $(r=0.49)$, and flexibility $(r=0.28)$ in healthy soldiers. ${ }^{19}$ Christoper et al. did not find a relationship between FMS and athletic performance. ${ }^{20}$ In several studies not yet used to assess subjects with CLBP in particular, there is no relationship investigated between disorder assessment tools such as the VAS, ODI, and back endurance tests. Therefore, the objective of this study is to determine whether FMS scores could evaluate the physical function of subjects with CLBP and to identify a relationship between the VAS and FMS, the ODI and FMS, and trunk endurance and FMS.

\section{METHODS}

\section{Subjects}

Twenty-nine subjects from 23-65 years of age participated in this study. All participants had LBP lasting more than 12 weeks with or without lower leg pains. Their mean age, height, and weight were $45.8 \pm 14.4$ years, $161.8 \pm 7.3 \mathrm{~cm}$, and $60.0 \pm 9.8 \mathrm{~kg}$ respectively. Exclusion criteria were subjects with neurological diseases, scoliosis, kyphosis, ankylosing spondylitis, shoulder pain, cancer, rheumatoid arthri- tis, and people who had ever undergone spinal surgery. The Institutional Research Review committee of Inje University gave ethics approval, and all participants reviewed and signed informed consent forms before volunteering.

\section{Procedures}

To assess physical function, FMS and back endurance tests were used. Pain level and disability were assessed using the VAS and ODI. ${ }^{3}$

The Sorensen test is used as method for testing isometric back endurance; it measures how long the subject can maximally maintain the unsupported trunk horizontally while lying prone with a fixed pelvis and, knees and ankles held by straps on a test table (Figure 1). ${ }^{8,9}$ The FMS includes seven tasks: a deep squat, hurdle step (right and left), active straight leg raise (right and left), rotary stability (right and left), inline lunge (right and left), push up, and shoulder mobility (right and left) (Figure 2). The test is rated on a 21point scale. If pain is perceived during task execution, a score of zero is assigned. ${ }^{2}$

\section{Statistical analysis}

The data of all the subjects were analyzed with descriptive statistics, and the results were reported as mean $\pm \mathrm{SD}$. The relationships between FMS and the other measurement tools were analyzed using Pearson's correlation coefficient.

Table 1. Demographic characteristics of subjects with NCLBP

\begin{tabular}{cc}
\hline Characteristics & Value \\
\hline Gender $(\mathrm{M} / \mathrm{F})$ & $7 / 22$ \\
\hline Age (years) & $45.8 \pm 14.4$ \\
\hline Height $(\mathrm{cm})$ & $161.8 \pm 7.3$ \\
Weight $(\mathrm{kg})$ & $60.0 \pm 9.8$ \\
\hline
\end{tabular}

Data are expressed as mean $\pm \mathrm{SD}$.

Abbreviations: NCLBP, nonspecific chronic low back pain; M, male; F, female.

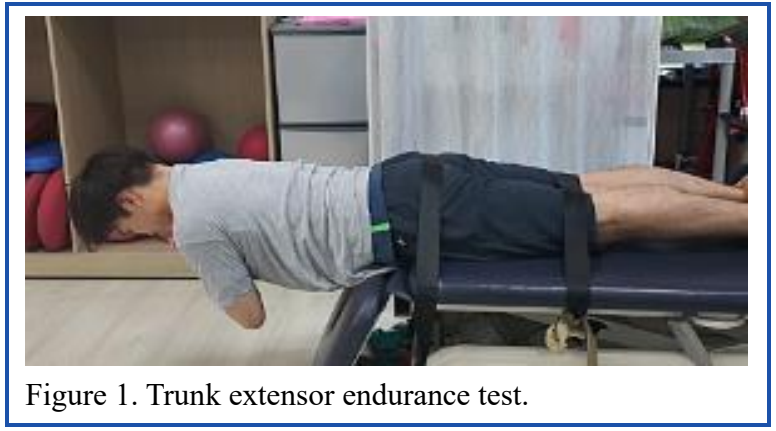



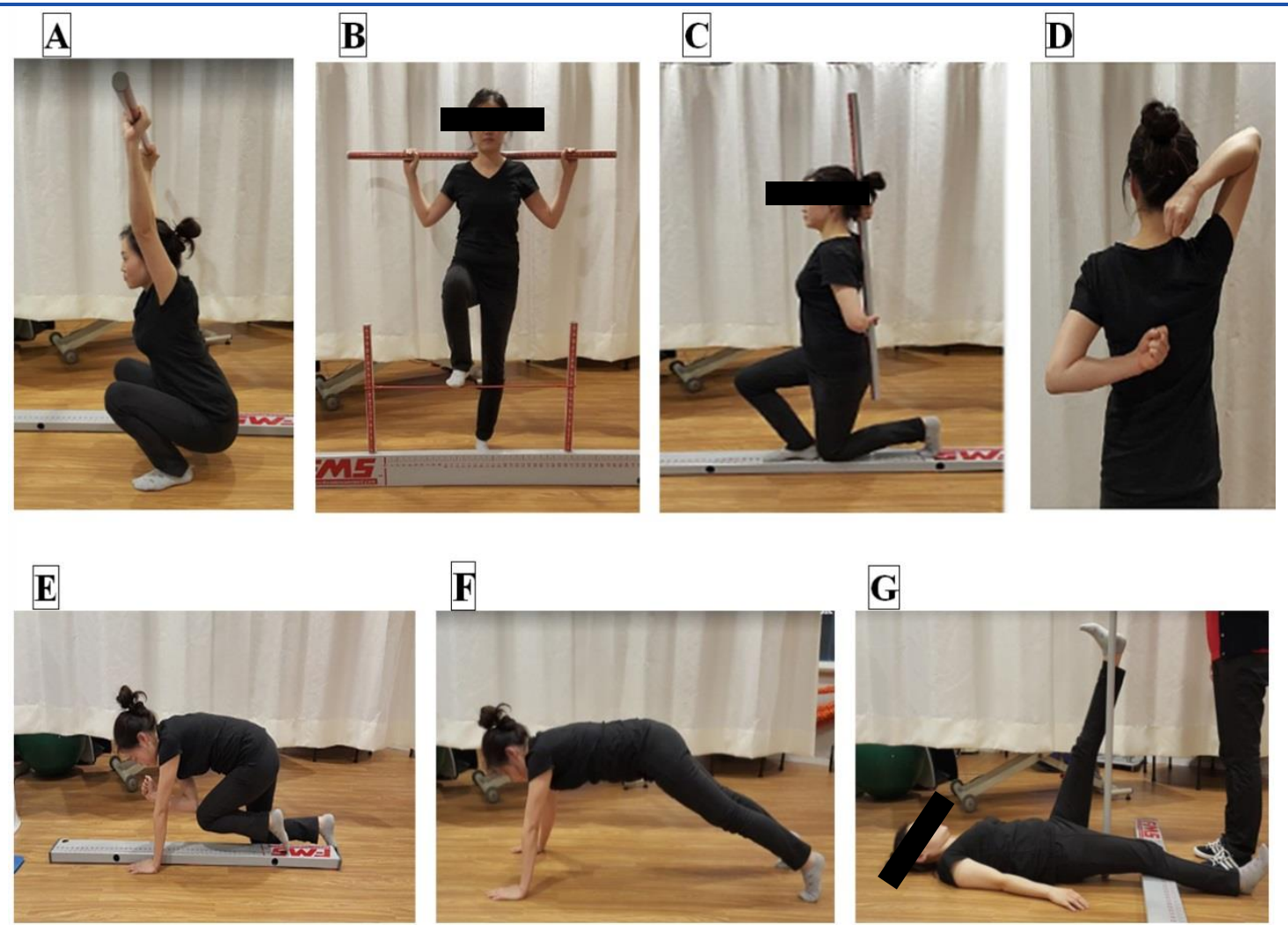

Figure 2. Seven tasks of functional movement screening (FMS).

(A) Deep squat, (B) Hurdle step, (C) Inline lunge, (D) Shoulder mobility, (E) Rotary stability, (F) Push-up, (G) Active straight leg raise.

The variables were assessed for outliers, and the criterion for an outlier was set at $>3$ standard deviations (SDs) from the mean value. Statistical Package for the Social Sciences (SPSS) ver. 18.0 (SPSS Inc., Chicago, USA) was used for analyses, and the significance was the adopted value of $p<0.05$.

\section{RESULTS}

FMS scores averaged 10.6. There was a statistically significant negative correlation between FMS and ODI scores ( $r=0.473, p<0.01)$, and the FMS and VAS scores $(r=0.409$, $p<0.05)$, respectively. The back endurance $(r=0.381, p<$ 0.041 ) positively correlated with FMS scores (Table 2) (Figure 3).

Table 2. Correlations between ODI, VAS, trunk extensor endurance test, and FMS

$(n=29)$

\begin{tabular}{cccc}
\hline & ODI & VAS & $\begin{array}{c}\text { Trunk extensor } \\
\text { endurance test }\end{array}$ \\
\hline FMS & $-0.473^{* *}$ & $-0.409^{*}$ & $0.381^{*}$ \\
\hline
\end{tabular}

$* p<0.05, * * p<0.01$.

ODI, oswestry disability index; VAS, visual analogue scale;

FMS, functional movement screening.

\section{DISCUSSION}

The primary purpose of this study is to determine the relationship between the ODI, VAS, trunk extensor endurance tests, and FMS in subjects with NCLBP, and the secondary purpose was to quantify and integrate their physical activity levels. We assessed physical functions that combined functional abilities through FMS. In the seven FMS tasks, active straight leg raise was used to assess the flexibility of the hamstring and the calf muscle, and push-up and rotary stability were used to assess trunk stability. Deep squat was used to assess the coordination of core stability and extremities, and the inline lunge and hurdle step were used to assess trunk stability, gluteal strength, proprioception, balance, and hip, knee, and ankle mobility. ${ }^{16}$

The total FMS score of all participants ranged from 7-16. The average was 10.62 of a possible total of $21,,^{2,21}$ Previous studies found a significant direct relationship between a history of LBP and decreased ASLR. ${ }^{19}$ Shum et al. reported limited motion in the hip and lumbar spine of subjects with CLBP during sit-to-stand and stand-to-sit motions. ${ }^{13}$ Sung et al. suggested significantly decreased kinetic and kinematic stability, poor balance performance, and an altered proprio- 

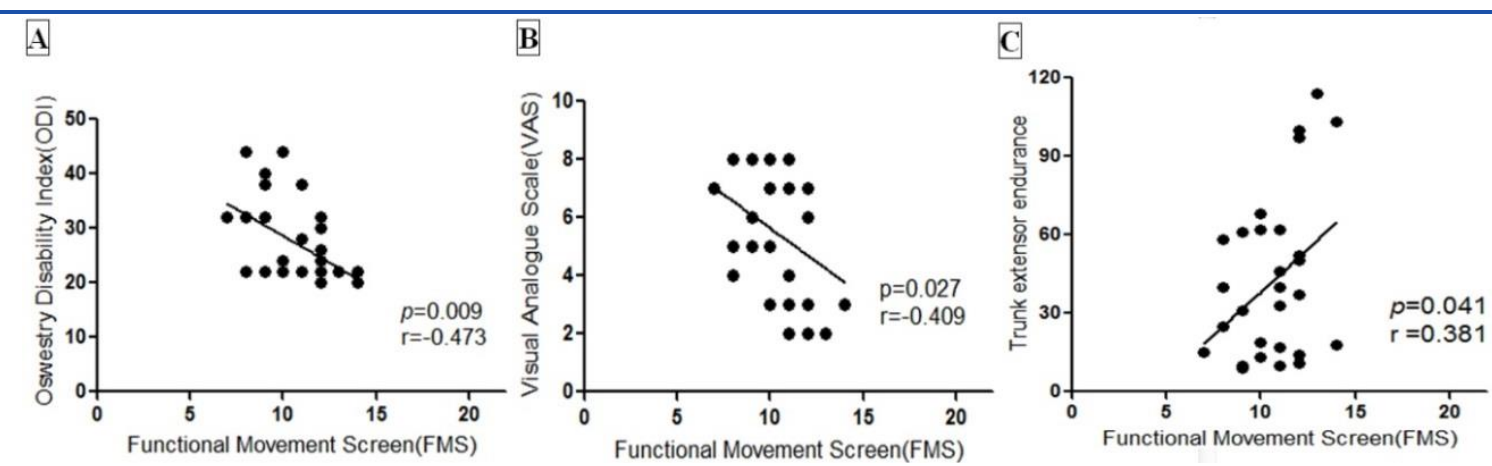

Figure 3. Correlation of ODI, VAS, trunk extensor endurance test, and FMS.

(A) Correlation of ODI and FMS scores, (B) Correlation of VAS and FMS scores, (C) Correlation of trunk extensor endurance and FMS score.

ceptive postural control during one leg standing exercises in subjects with CLBP. ${ }^{22}$ Other studies reported decreased spinal mobility, endurance, strength, and physical fitness in the CLBP population. ${ }^{23-25}$ In our study, low total FMS scores may be associated with deficit movement or disability in subjects with CLBP. 2,15

There was a statistically significant relationship between back endurance times and FMS scores $(r=0.381)$. Possible reasons for these results may be poor back extensor muscle endurance and impaired body movement and coordination. Back endurance was measured as the total time subjects were able to maintain an unsupported trunk on a horizontal examination table. Trunk muscles play an important role in supporting and stabilizing the lumbar spine. ${ }^{26,27}$ Poor back extensor muscle endurance is related to a low fatigue threshold ${ }^{1}$ and causes uncontrollable trunk movement. ${ }^{26}$ Mitchell et al. found that total FMS scores were associated with core strength and dynamic posture. ${ }^{18}$ FMS measured trunk stability through push-ups, rotary stability, deep squats, inline lunges, and hurdle steps. ${ }^{2,15,16}$ Based on the results of the current investigation, FMS is a useful tool for determining and integrating the functional movement capabilities of the population with CLBP and could add valuable information for subjects with CLBP. We believe that FMS may assist to design more effective individualized treatment plans to improve the functional capacity of subjects with CLBP.

We found a statistically significant but small $(r=0.41)$ negative correlation between VAS and FMS scores. There was also a negative $(r=-0.473)$ correlation between ODI and FMS scores. The ODI score was measured as the total score in a questionnaire about the functional status and pain involved in daily activities. Subjects with CLBP have decreased postural control, spinal mobility, endurance, strength, and physical fitness. ${ }^{23-25}$ Originally, it was deve- loped as a screening tool for football players ${ }^{17}$ and predicted their injuries if their scores were 14 or less. $^{2}$ Our results should be significant for the clinical setting because FMS averaged approximately 10 . Our results may be clinically meaningful for subjects with NCLBP, as it may assist the evaluation of their physical functions. Further, the relationship between self-reported (ODI and VAS) and clinical measures (FMS) may assist in the design of preventive programs associated with decreased functional movement.

However, our results did not confirm the results of leftright asymmetry nor calculate the score of each task. Therefore, we will not provide information about specific disabilities nor the functional characteristics of participants. In addition, the sample size was small in this study. Further research is needed to compare FMS scores between healthy subjects and subjects with LBP.

\section{CONCLUSIONS}

Our study identified the relationship between trunk endurance and VAS or ODI and FMS. The results showed a small negative correlation between VAS score and FMS score. There was also a negative correlation between ODI and FMS. There was a positive relationship between back endurance time and FMS. Therefore, FMS may assist in designing more effective individualized treatment plans to improve the functional capacity of subjects with CLBP.

\section{Key Points}

Question Can FMS scores evaluate the physical function of subjects with CLBP and to identify a relationship between the VAS and FMS, the ODI and FMS, and trunk endurance and FMS? 
Findings There was a statistically significant negative correlation between FMS and ODI scores, and the FMS and VAS scores, respectively. The back endurance positively correlated with FMS scores.

Meaning FMS may assist in designing more effective individualized treatment plans to improve the functional capacity of subjects with CLBP.

\section{Article information}

Conflict of Interest Disclosures: None.

Funding/Support: None.

Acknowledgment: None.

\section{REFERENCES}

1. Mayer TG, Gatchel RJ, Kishino N, et al. Objective assessment of spine function following industrial injury. A prospective study with comparison group and one-year follow-up. Spine. 1985;10(6):482-493.

2. Cook G, Burton L, Hoogenboom B. Pre-participation screening: The use of fundamental movements as an assessment of function-part 1. N Am J Spots Phys Ther. 2006;1(2):62-72.

3. Sánchez-Zuriaga D, López-Pascual J, Garrido-Jaén D, de Moya MF, Prat-Pastor J. Reliability and validity of a new objective tool for low back pain functional assessment. Spine. 2011;36(16):1279-1288.

4. Fairbank JC, Couper J, Davies JB, O'Brien JP. The oswestry low back pain disability questionnaire. Physiotherapy. 1980;66(8):271-273.

5. Grönblad M, Hupli M, Wennerstrand P, et al. Intercorrelation and test-retest reliability of the Pain Disability Index (PDI) and the Oswestry Disability Questionnaire (ODQ) and their correlation with pain intensity in low back pain subjects. Clin J Pain. 1993;9(3):189-195.

6. Spratt KF, Lehmann TR, Weinstein JN, Sayre HA. A new approach to the low-back physical examination: Behavioral assessment of mechanical signs. Spine. 1990;15(2): 96-102.

7. Coorevits P, Danneels L, Cambier D, Ramon H, Vanderstraeten G. Assessment of the validity of the BieringSørensen test for measuring back muscle fatigue based on EMG median frequency characteristics of back and hip muscles. J Electromyogr Kinesiol. 2008;18(6):997-1005.

8. Sorensen BF. Physical measurements as risk indicators for low-back trouble over a one-year period. Spine. 1984; 9(2):106-119.

9. Latimer J, Maher CG, Refshauge K, Colaco I. The relia- bility and validity of the Biering-Sorenson test in asymptomatic subjects and subjects reporting current or previous nonspecific low back pain. Spine. 1999;24(20):20852089.

10. Arokoski JP, Kankaanpää M, Valta T, et al. Back and hip extensor muscle function during therapeutic exercises. Arch Phys Med Rehabil. 1999;80(7):842-850.

11. Demoulin C, Vanderthommen M, Duysens C, Crielaard JM. Spinal muscle evaluation using the Sorensen test: a critical appraisal of the literature. Joint Bone Spine. 2006;73(1):43-50.

12. Ng JK, Richardson CA, Jull GA. Electromyographic amplitude and frequency changes in the iliocostalis lumborum and multifidus muscles during a trunk holding test. Phys Ther. 1997;77(9):954-961.

13. Shum GL, Crosbie J, Lee RY. Effect of low back pain on the kinematics and joint coordination of the lumbar spine and hip during sit-to-stand and stand-to-sit. Spine. 2005;30(17):1998-2004.

14. Sung PS, Yoon B, Lee DC. Lumbar spine stability for subjects with and without low back pain during one-leg standing test. Spine. 2010;35(16):E753-760.

15. Cook G, Burton L, Hoogenboom B. Pre-participation screening: The use of fundamental movements as an assessment of function-part 2. N Am J Spots Phys Ther. 2006;1(3):132-139.

16. Cook, G. Movement: Functional movement systems: screening, assessment, and corrective strategies. Santa Cruz: On Target Publications; 2010.

17. Kiesel K, Plisky P, Butler R. Functional movement test scores improve following a standardized off-season intervention program in professional football players. Scand J Med Sci Sports. 2011;21(2):287-292.

18. Mitchell UH, Johnson AW, Adamson B. Relationship between functional movement screen scores, core strength, posture, and BMI in school children in Moldova. J Strength Cond Res. 2015;29(5):1172-1179.

19. Teyhen DS, Shaffer SW, Lorenson CL, et al. Clinical measures associated with dynamic balance and functional movement. J Strength Cond Res. 2014;28(5):12721283.

20. Parchmann CJ, McBride JM. Relationship between functional movement screen and athletic performance. $J$ Strength Cond Res. 2011;25(12):3378-3384.

21. Ibrahim AI, Muaidi QI, Abdelsalam MS, Hawamdeh ZM, Alhusaini AA. Association of postural balance and isometric muscle strength in early-and middle school age boys. J Manip Physiol Ther. 2013;36(9):633-643.

22. Sung PS, Yoon B, Lee DC. Lumbar spine stability for 
subjects with and without low back pain during one-leg standing test. Spine. 2010;35(16):E753-760.

23. Cleland J, Gillani R, Bienen EJ, Sadosky A. Assessing dimensionality and responsiveness of outcomes measures for subjects with low back pain. Pain Pract. 2011; 11(1):57-69.

24. Parks KA, Crichton KS, Goldford RJ, McGill SM. A comparison of lumbar range of motion and functional ability scores in subjects with low back pain: assessment for range of motion validity. Spine. 2003;28(4):380-384.

25. Wittink H. Functional capacity testing in subjects with chronic pain. Clin J Pain. 2005;21(3):197-199.

26. Parianpour M, Nordin M, Kahanovitz N, Frankel V. The triaxial coupling of torque generation of trunk muscles during isometric exertions and the effect of fatiguing isoinertial movements on the motor output and movements patterns. Spine. 1988;13(9):982-992.

27. Takemasa R, Yamamoto H, Tani T. Trunk muscle strength in and effect of trunk trunk muscle exercises for subjects with chronic low back pain. Spine. 1995;20 (23): 2522-2530. 